\title{
The Enlightenment of the Interconnection between the Internet Backbone Networks in the United States to China
}

\author{
Meijuan $\mathrm{Li}^{\mathrm{a}}$, Qianbing Xiao ${ }^{\mathrm{b}}$

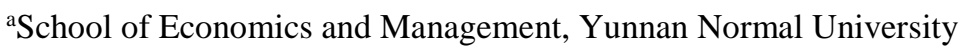 \\ Kunming and 650500, China \\ ${ }^{\mathrm{b}}$ School of Economics and Management, Yunnan Normal University \\ Kunming and 650500, China
}

\begin{abstract}
The Internet, as a network, is composed of multiple interconnected networks. Interconnection between networks is the basic premise of the existence of Internet. Since the Internet was introduced into China, it has been developing for more than 20 years. The Internet backbone networks has evolved from one to multiple, and the issue of interconnection has also arisen during the development process. How to effectively manage the interconnection of networks has become a problem that the government needs to be addressed urgently. Through the analysis of the development process of the Internet backbone networks in the United States, this paper summarizes the interconnection experience between the Internet backbone networks in the United States, and combines the actual development of backbone networks in China to explore the useful enlightenment and suggestions for the interconnection of the internet backbone networks in China.
\end{abstract}

Keywords-Internet backbone network; Interconnection; Interconnection structure; Interconnection mode

\section{INTRODUCTION}

The United States is the origin of the Internet. After 50 years of development, it has entered into a relatively mature stage, which plays a leading role in the development of the Internet industry and has always been a reference model for other countries. Similarly, the interconnection experience of Internet backbone in United States has also played an important role for the China's Internet backbone development.

\section{THE DEVELOPMENT STAGE OF INTERCONNECTION} BETWEEN THE INTERNET BACKBONE NETWORKS IN THE UNITED STATES

The development of Internet backbone interconnection in the United States can be divided into the following three stages:

\section{A. The initial development period of interconnection}

In the late 1960s, the U.S. Department of Defense established a network called ARPANET. In the mid-1980s, the National Science Foundation (NSF) took over the ARPANET and established the National Science Foundation Network (NSFNET), which has a transmission rate of $56 \mathrm{kbit} / \mathrm{s}$. In 1987 , Merit, IBM and MCI began to operate NSFNET together. At this time, the network which supervised by government was only used for academic research, which was forbidden to be applied in commercial fields. NSFNET was the only one Internet backbone in the United States at that time, so there was not exist interconnecting problems between networks.

\section{B. The starting period of interconnection}

Subsequently, the commercial network emerged and developed rapidly. At the end of the 1980s, the US strictly prohibited any related business activities on the National Science Foundation Network (NSFNET), which hindered commercial networks from exchanging information with other networks connected to NSFNET. In order to remove this development obstacle, some commercial networks have jointly established a commercial network exchange system for the traffic exchange between end users. In 1993, the National Science Foundation began to commercialize and Network Access Point (NAP) emerged. Eventually, the commercial network and NAP replaced NSFNET in 1995.

However, because the NAP did not limit the number of backbone networks connected to it, it also be called public peering. The more and more backbone network enterprises connected to the NAP, which intensified the congestion. In addition, the mismatch between geographic location of NAP and backbone networks resulted into high bandwidth cost, long transmission time and poor quality, which made the mode of peering emerge [1]. The interconnection modes of backbone network in the US are mainly Bill-and-keep peering, which mainly occurs between backbone networks with similar network capacity and user scale. Moreover, the two parties do not settle each other in this mode. 


\section{The mature period of interconnection}

For a long time, it is free for the vulnerable backbone networks that no matter which interconnection mode they adopt. The large-scale backbone network pays more costs than a small one, but it gains less profits. Meanwhile, it needs to compete with the small backbone network in the user market.

The expansion incentives for the large-scale networks will reduce. Even if they are connected to the small, the interconnection quality will be at a low level. The unfair interconnection mechanism makes that the large-scale backbone networks begin to establish new and higher bill-andkeep peering standards, and networks that do not meet the standards will be eliminated [2]. At this point, the new interconnection mode - transition has come into being. The backbone network that does not meet the requirements of the large networks will access to the Internet by transition.

At present, there are three types of interconnection mode in the United States: public peering, bill-and-keep peering and transit, in which the most important mode are the latter two. The six top backbone network which located on the first tier of the US backbone network structure: AT\&T, Sprint, UUNet, Qwest, C\&W and Level3 generally exchange traffic through bill-and-keep peering. The second tier are national backbone networks. Due to the difference in scale and development level, they can only access to the Internet through transit and paid peering. The third tier are regional backbone networks. Similar to the second tier, interconnection of backbone networks in different tier can only through transit, while the backbone networks at the same tier are exchanged traffic through paid peering [3].

\section{THE INTERCONNECTION EXPERIENCE OF INTERNET BACKBONE NETWORK IN THE UNITED STATES}

In the United States, the Internet backbone network has gradually matured after years of development. During this period, a complete set of interconnection systems was formed, and a great deal of interconnection experience are also accumulated during the development.

\section{A. The backbone network structure is obvious and competition between networks is fierce}

At present, the backbone network market in US is divided into three tiers based on the size. There are a large number of backbone networks, in which, the size of backbone networks in the same tier is the same [4]. They are interconnected with each other through bill-and-keep peering and freely exchange traffic. While there is still a huge gap in the size of the networks with the different tier, the interconnection between them is mainly through transit and paid peering. Under the opening of the international gateway, the low-tier backbone network can also bypass overseas to purchase transit services, which achieves interconnection with high-tier backbone networks so as to make the backbone network fully competitive.

In the US, the Internet backbone networks in the same tier interconnect each other through bill-and-keep peering, which eliminates the cumbersome settlement process and improves the operational efficiency. The backbone network in the second and third tier can not reach a bill-and-keep peering protocol with the Tier-1, but it can always access to the Internet at a lower price. First, all the backbone network mainly obtain revenue by selling transit services to the low-tier backbone network. The Interconnection mode of transit makes the US backbone network market an upstream-downstream relationship between service providers and customers.

Each large network wants to get more customers and income through transit, which makes the competition fierce, as well as homogeneity product features of transit, so the price level has reduced. Second, in the interconnection mode of paid peering, the backbone networks which in the high-tier only needs to provide traffic exchange services without signing a service-level agreement (SLA), which has less responsibility than the transit. Therefore, if the backbone networks are interconnected by paid peering, they will pay less than the transit. Finally, the settlements for the transit and paid peering are also affected by the international transit price. The prices under the three interconnection mode are mutually restrictive, with the result that the interconnection quality between the backbone network of the United States has been improved, the settlement fee has been reduced, and the social welfare has been improved.

\section{B. The interconnection between backbone networks is determined by the market}

The U.S. government has always adopted the policy of "laissez-faire" for the interconnection between Internet backbone networks, which means that the interconnection mode and settlement between the backbone networks are decided by themselves. It is the result of free competition in the market. The government does not intervene, but it hopes to promote technological progress and industrial development of the Internet through competition. The Internet structure in the US, the backbone network has formulated a harsh condition for bill-and-keep peering, which refuses to establish a relationship of bill-and-keep peering with the vulnerable backbone network, but provide services of transit and paid peering for them. This not only saves resources and improves efficiency, but also avoids the behavior of "free riders". The more important is that they can obtain a part of revenue from transit and paid peering, form a situation of full competition. In terms of interconnection settlement, the settlement fee is spontaneously determined by the market. In the United States, the settlement fee is mutually restricted with a variety of interconnection mode [5]. There is no incentive to increase the settlement fee in any kind of interconnection mode. Under the impetus of this restriction mechanism, each backbone network has improved its own efficiency, which make the interconnection settlement fee finally remain a relatively low level.

It is worth noting that the premise of the policy of "laissez faire" in the US is that the market is free competition. In the United States where the legal system is perfect, the backbone networks must be operated within the scope of the "anti-trust act" and "competition law", which are mainly designed to restrict the emergence of the dominant backbone network in the market and prevent the abuse of market power by largescale backbone networks, and hinder the development of the small-scale backbone networks. 


\section{NAP interconnection is a mode of "sender keep all" and the costs of operators are reduced}

NAP interconnection is the earliest interconnection mode in the United States. In the early stage of the commercialization of the Internet, the numbers of backbone network were relatively small and the development levels were not different. They were easy to reach a multilateral bill-and-keep peering protocol, and the backbone network connected the NAP did not mutually pay any settlement fee, in addition to shoulder the cost of the transmission broadband and relative equipment for the interconnection. The NAP exists as a public good. With the development of the Internet, more and more Internet backbone networks, ISPs, and ICPs are connected to the NAP, which often leads to congestion, packet loss and time-delay. That solely relying on the NAP interconnection mode to exchange traffic between networks can not meet the requirements of those operators, many backbone operators begin to interconnect with other operators through a new mode---billand-keep peering. The backbone networks that interconnected by the mode of bill-and-keep peering are of similar size. As same as the NAP, the mode of bill-and-keep becomes widely used in peering. In the current structure of the US internet backbone network market, the backbone network which similar in size usually establish a relationship of bill-and-keep peering [6]. At this point, they are able to not only avoid to pay settlement fees in NAP interconnection mode, but also in the mode of Bill-and-keep peering. The NAP interconnection exists as a complement of other interconnection mode. This complementary effect saves costs and reduces the cost pressure of the vulnerable backbone network, which make the vulnerable backbone network become competitiveness in the backbone network market.

\section{The main interconnection mode between backbone networks is Bill and Keep peering}

When the backbone network began to be commercialized, the NAP interconnection was the only way for the interconnection of the backbone network in the United States. It succeeded in promoting the interconnection of the Internet backbone network in the US, but in the long run, it was not a successful mode. Now there are only a few NAP or traffic exchange points in the US, and they may not match the geographic scope that backbone network can reach. For example, most backbone networks have interconnection nodes in cities such as New York and Boston, but the nearest NAP is in New Jersey. Therefore, when the traffic of a backbone network flows from Boston to other backbone networks of the same city, it is necessary to complete this transmission through the NAP in New Jersey, which greatly reduces the transmission efficiency, and the traffic is easily delayed in the transmission process.

Network operators investing in high-capacity links that access NAP need to bear the total cost of the investment, but they can only receive a portion of the revenue. This asymmetry of investment and earnings hinders the network operator's to upgrade NAP link capacity. In addition, due to the lack of traffic-based pricing mechanisms in NAP, networks often excessively use links that connected to the NAP, making excessive congestion of the NAP. The two problem make the
NAP unable to meet the interconnection requirements of many large-scale networks, so that the bill-and-keep peering and transit have been well developed. At the beginning of the development of the Internet backbone network in the United States, the size of the backbone networks is not much different. Nowadays, the interconnection between networks has matured, and the scale of backbone networks has begun to differ. However, there are still a large number of backbone networks similar in size. Most of the Internet traffic in the United States is transmitted through bill-and-keep peering. Traffic transmission in this mode is convenient and fast, which does not involve network settlement. Now, only a small portion traffic transmit through transit and paid peering.

\section{THE ENLIGHTENMENT OF THE INTERCONNECTION BETWEEN INTERNET BACKBONE NETWORKS IN THE UNITED STATES TO CHINA}

As the birthplace of the Internet, the United States has become the country with the largest number of Internet users, and the overall strength of its backbone is best in the world. This section will combine the China's development and draw experience from the development of Internet backbone network in the United States, so as to get some enlightenments from the U.S.

\section{A. The competitive market structure is the key to solve the dilemma of the interconnection between backbone networks}

At present, there is a phenomenon of "the north-south divide and conquer" with backbone network market in China. The China Telecom and China Unicom are powerful and dominate the north and south markets. Any decision-making they make in the interconnection will affect other backbone networks in the market. On the one hand, it is extremely prone to produce the coexistence of high settlement fees and low interconnection quality. On the other hand, the dominant backbone networks may extend its monopolistic power to the downstream ISPs market in the context of regional monopoly, and bring huge losses to the downstream ISPs. Looking back on the Internet backbone market in the United States, a large and layered market structure of backbone networks which formed in the process of development makes the market an in

Without any mandatory force of the government, the market can spontaneously form a fully competitive situation. When a backbone network is unwilling to provide interconnection service for low-level backbone networks or increase the settlement fee, the low-level backbone networks can look for other options that establish paid peering or transit relationship with high-tier backbone networks. The quality of interconnection will increase in competition and the satisfaction of Internet users will increase. Therefore, according to the experience of the interconnection between backbone networks in the United States, the key resolving the predicament of backbone network interconnection is to change the existing structure of the backbone network market and form a reasonable and effective market competition situation. 


\section{B. The coexistence of various interconnection modes is conducive to promote the competition of backbone networks}

Since the backbone network developed in China, there are only two interconnection modes: NAP and peering. This not only limits the interconnection choices of the backbone network, but also increases the operating costs of the access party, which is not conducive to the formation of competition between networks. In particular, the paid peering mode adopted by vulnerable backbone network and leading backbone network in China has a severe anti-competitive feature. First, due to the lack of enthusiasm for interconnection of large-scale backbone networks, the small-scale backbone network can only pay a certain fee for the large backbone network to encourage them to provide high interconnection quality. While the largescale backbone network is in a competitive position, it is likely to charge too much for the small backbone networks and increase their cost, which makes the competitiveness of the small-scale further weaken and widen the gap between the two sides. Second, the paid peering has weakened the competition between the two leading backbone networks in China. Because China prohibits transit between backbone networks, vulnerable backbone networks must respectively establish a relationship of paid peering in order to exchange traffic with the two major backbone networks. At this time, the two leading backbone networks do not have to compete for the access of the vulnerable backbone networks, and each of them can maintain their respective market power in the backbone network market.

From the interconnection experience of the Internet backbone network in the US, it can be seen that the interconnection between backbone networks of different sizes can also adopt transit interconnection in addition to paid peering. Under the transit, the vulnerable backbone networks only needs to buy the transit service of the high-tier backbone network to access to the Internet, which reduces the interconnection cost and improves the interconnection efficiency. At this time, the backbone networks that provide transit services also compete for customers. In addition, both the buyer and the seller of the transit service must sign a "service-level agreement" when they reach a purchase agreement, which ensures the quality of interconnection and makes up for the shortcomings of low quality and poor performance of peering in our country.

\section{The legislation of Internet is the key to interconnection of internet backbone}

The behavior norm of the US backbone network operators has always been guided by laws. The government has always adopted an unregulated attitude and the market is free to compete. But the Internet backbone network market structure in China is different of the United States, and its development is still immature. The market cannot be completely regulated by economic means. It must join the government's administrative power to promote development. Without government regulations, the vulnerable backbone network operators do not have the possibility to compete fairly with China Telecom and China Unicom, nor do they have the strength to compete with the two. Coupled with the fact that Internet backbone network cannot provide transit services in
China, the vulnerable backbone network have to pay high settlement fees for paid peering, which seriously affects the competitiveness of the vulnerable backbone network. In addition, disputes over the unilateral settlement have always existed in China, and a series of problems in the interconnection show that the internet legislation is indispensable at this stage.

The perfect laws system is also the basis for government regulation. Compared with China, the United States is the country where the Internet was born, and the internet has developed for more than 20 years. At present, the United States has formed a relatively complete Internet legal system. The advancement of the legislative work make the Internet management system normally operate. In China, the Internet legislation is also important. However, the legal system of Internet in China is not perfect at this stage, and the problems in interconnection often have no corresponding laws, which leads to "no law to follow". Therefore, the emphasis of interconnection in the future should be placed on government regulation and legislation.

\section{The interconnection system suitable for our country is the guarantee for the success of interconnection}

In the early stage of the development of the Internet in the United States, the scales of the backbone network operators are not very large and not much different, which makes the early American interconnection mainly focused on bill-and-keep peering. With the development of the Internet, many new backbone networks have appeared in the market. They generally access to the Internet through paid peering and transit. So far, the backbone network market in the US has coexisted in three interconnection modes and formed a hierarchical market structure [7]. Among them, bill-and-keep peering is the most important interconnection mode at different tiers. With full competition at all tiers of the network and the well-established Internet legal system in the United States, the government does not basically need to supervise the backbone network market.

Looking back on the development of Internet backbone network market in China, in the early stage of the commercialization of the Internet, there was only a backbone network of China Telecom in the market, and the network covered the whole country. Later, although some other backbone network enterprises have been formed in the market, in terms of scale and competitiveness, these backbone networks are far behind China Telecom and China Unicom that was later split and reorganized[8].Therefore, the interconnection mode based on bill-and-keep peering is often difficult to spontaneously form in China. The monopoly power of the leading backbone network is strong, and the laws of Internet interconnection are not well established. If the government does not regulate the backbone market at this time, it is bound to intensify the monopoly of the leading network, and the vulnerable backbone networks are also hard to develop. According to the analysis above, the development of the backbone network in China can not completely copy the experience of the United States. It is necessary to analyze the current situation of the backbone network market in China, daw lessons from the good development experience of the 
United States to search for an interconnected system suitable for the development of backbone network in China.

\section{CONCLUSION}

The research has obtained: the multi-level backbone network structure, the interconnection of marketization methods, the bill-and-keep between NAP points, and the peering method are valuable experience in the development of Internet backbone network in the US, which has created the power of the American Internet. The development of China's Internet backbone network can learn from the relevant experience of the development of the backbone network in the US. The key is to change the existing market structure of China's backbone network market, introduce transit interconnections on the basis of existing interconnection methods, and form the interconnection patterns of multiinterconnection mode coexistence. Strengthen Internet legislation and regard it as the focus of interconnection and intercommunication. The interconnection system that suits the China's actual development is the guarantee for the success of China's backbone network interconnection.

\section{REFERENCES}

[1] Cremer J, Rey P and Tirole J. Connectivity in the Commercial Internet [J]. Journal of Industrial Economics, 2000, 48(4):433-472.

[2] Wei Liurong, Wang qi; Lv Tingiie. The Development Situation of American Internet Inter-working and its Revelation to China [J].World Telecommunications, 2005, (05):15-19. (In Chinese)

[3] Zhao Liang, Yang Hua. Analysis and Suggestions on the Interconnection of Internet Backbone Network in China [J].Communications World, 2005, (28):40. (In Chinese)

[4] Wei Liurong, Wang qi; Lv Tingjie. Revelation of interconnection in the United States [J]. China Telecommunications Trade, 2005(03):68-70. (In Chinese)

[5] Ding Ke. Analysis of Internet Backbone Market Problems and Policy Suggestions in China [J]. Guangdong Communication Technology, 2007, (06):10-14. (In Chinese)

[6] Zhang Bin, Chen Siqi, Yang Hai. International General Mode of Internet Backbone Network Interconnection [J]. Communications World, 2007, (22):25-26. (In Chinese)

[7] Zeng Jianqiu, Yin Jinyi, Yu Yi. Pay attention to Internet interconnection [J]. Jiangsu Communication, 2009, 25(05):32-35. (In Chinese)

[8] Zhang Xinzhu, Chen Jian. Methods of Settlement Governance for Internet Backbone: Comparing Chinese and International Models [J]. Comparative Economic \& Social Systems, 2013, (03):179-188. (In Chinese) 